\title{
Using combinations of spatial gradients to improve the detectability of buried conductors below or within conductive material
}

\author{
Richard S. Smith ${ }^{1}$
}

\begin{abstract}
The detection of conductive bodies is an important capability when exploring for massive sulfide deposits or looking for unexploded ordnance. When these bodies are buried below conductive overburden or embedded in conductive material, the use of an electromagnetic system to identify the bodies becomes problematic because the response of the overlying conductive material can be much greater that the response of the buried conductor. I calculated the response of five models representing different conductivity distributions (a buried conductor, a uniform overburden with changes in the system altitude, a paleochannel, a thicker overburden, and a thinner overburden). The subtle response of the buried conductor was difficult to identify because it looked very similar to the responses of other structures that are not necessarily of interest. The spatial gradients for the same five models showed that the greatest improvement in the relative size of the anomalous gradient response compared with the
\end{abstract}

background gradient came for the cases in which the material closest to the surface changes, in particular the paleochannel and thickening overburden models. However, identification of the deeper buried conductor was still problematic because of the large background gradients. In theory, the cylindrical symmetry of a dipole transmitter over a layered earth ensured that there were exact relations between the spatial derivatives. Hence it was possible to define two specific combinations that should be zero over a layered earth. Calculating these combinations for the five models showed that the anomalous zones stood out with significantly greater anomaly-to-background ratios. The measurement of the gradients and the calculation of these combinations therefore provided a means of identifying anomalous zones in and below a conductive earth. Different relative sizes and shapes of the two combinations for different models provided a way of discriminating between the vertical conductor model and the four other models.

\section{INTRODUCTION}

The measurement of spatial gradients is well established in gravity and magnetic methods. The torsion balance, invented by Roland, Baron Eötvös of Vásárosnamény more than 100 years ago, measured a spatial gradient of the gravity field (Telford et al., 1976). Measurements of gradients have also been used in ground and airborne magnetometry (Telford et al., 1976; Hood and Teskey, 1989). Over the past 10 years, the introduction of airborne gravity gradiometry (van Leeuwen, 2000; Dransfield, 2007) has renewed interest in gradient measurements.

Sattel and Macnae (2001) undertake a synthetic study to investigate the feasibility of taking electromagnetic (EM) gradient measurements for an active EM system. They find that the gradient information was most useful for determining the shallow conductivity structure and providing lateral resolution. However, they conclude that the gradients had a limited capability for detecting deep vertical conductors.

The use of spatial and temporal gradients is advocated for estimating the conductance of a thin sheet (Kolaj and Smith, 2012) in a manner analogous to how the ratio of the measured electric and magnetic fields can be used for estimating the conductivity in plane-wave EM methods (Vozoff, 1991). This is not surprising because the spatial gradients are required to calculate the curl of the magnetic field, which is related through Ampere's law to the electric field in a conductive media. Some inductive EM systems measure the electric field directly: either using grounded electrodes (Macnae, 1981) or capacitive electrodes (Adams et al., 2010).

EM gradients are also advocated for an airborne EM system by Lee (2010), who argues that measuring the magnetic field and its

Manuscript received by the Editor 11 June 2012; revised manuscript received 30 August 2012; published online 20 December 2012.

${ }^{1}$ Laurentian University, Department of Earth Sciences, Sudbury, Ontario, Canada. E-mail: rssmith@laurentian.ca.

(C) 2012 Society of Exploration Geophysicists. All rights reserved. 
spatial gradients can be used to detect extremely conductive bodies. The key to Lee's (2010) approach appears to be using the gradients to estimate the geometry so that the primary can be calculated and then subtracted from the measured primary to yield a residual that may be due to an extremely conductive body.

The contribution of this paper is to show how spatial gradients, when combined, can be used to suppress the component of the response that is cylindrically symmetric (the overburden or layered half-space response) and identify and discriminate subsurface anomalous zones. The EM system is a separated dipole system; it can be a ground or an airborne EM system.

\section{THE PROBLEM OF CONDUCTIVE GROUND}

The problem of identifying conductive bodies using a separated dipole system is illustrated with a traditional fixed-wing airborne EM system. The transmitter is a vertical dipole of unit dipole moment, and there are receivers towed $130 \mathrm{~m}$ behind and $50 \mathrm{~m}$ a)

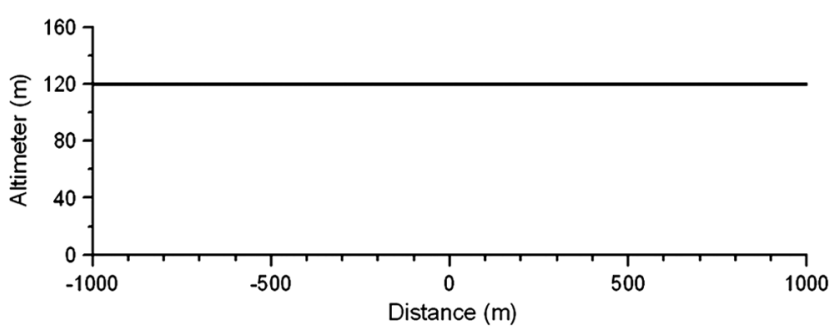

b)

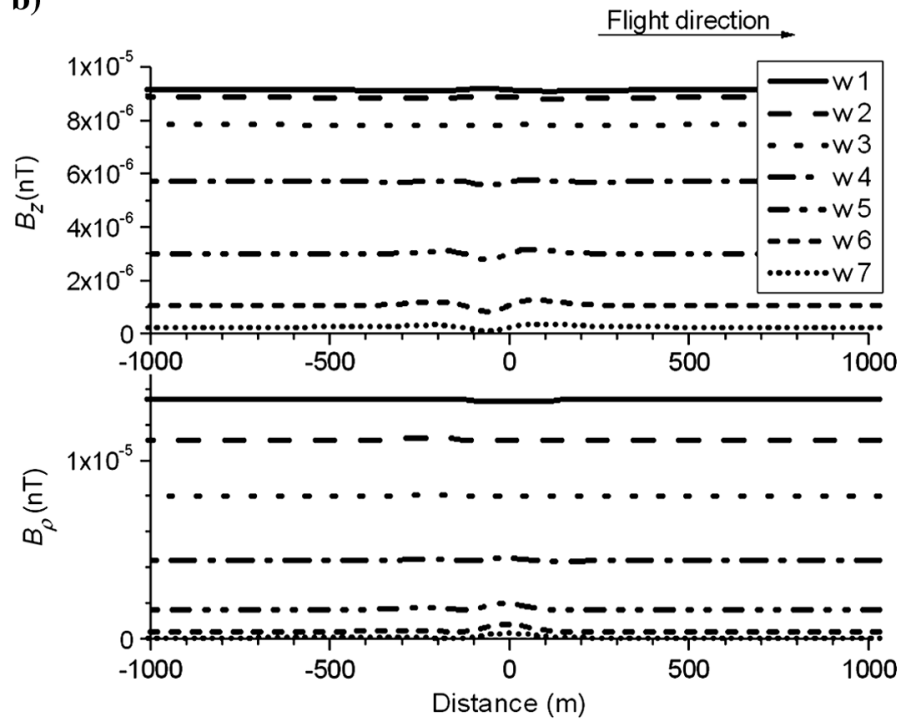

Figure 1. The geoelectric model of a vertical conductor of conductance $20 \mathrm{~S}$ with a 1000-m strike extent and a $500-\mathrm{m}$ dip extent, buried in $1000-\Omega \mathrm{m}$ material below a conductive overburden of $5 \Omega \mathrm{m}$ and thickness $60 \mathrm{~m}$. The airborne EM system is a vertical-dipole transmitter, a receiver towed $50 \mathrm{~m}$ below and $130 \mathrm{~m}$ behind the transmitter, measuring the vertical $z$ - and horizontal $\rho$-component responses. (a) The altitude of the aircraft is shown at the top, and it is $120 \mathrm{~m}$ across the whole profile. (b) The $z$ - and $\rho$-component responses are shown in the top and bottom panels, respectively. The details of the transmitter moment, the current waveform, and time windows W1 to W7 are given in the text.

a)

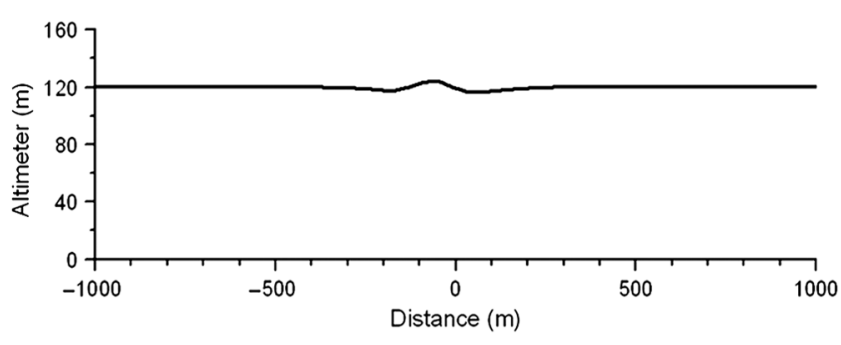

b)

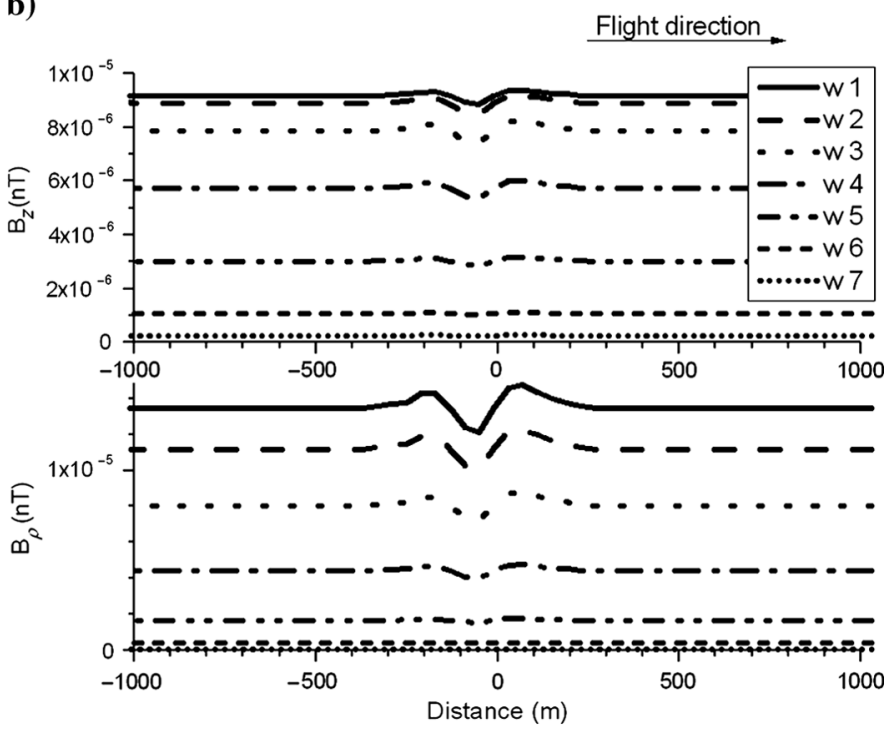

Figure 2. The same as Figure 1, except there is no vertical conductor and the height of the transmitter varies along the profile with the minimum value being $116.4 \mathrm{~m}$ and the maximum value $124.4 \mathrm{~m}$. Note that the shape of the anomalous response on the $z$-component at late time is similar to that in Figure 1. 
below the aircraft. In the simulations presented here, the system measures the vertical $(z)$ and horizontal $(\rho)$ components. EMIGMA modeling software (Murray et al., 1999) is used to calculate these components. The waveform used has a $30-\mathrm{Hz}$ base frequency, a $50 \%$ duty cycle current pulse with a $0.5 \mathrm{~ms}$ switch off, and seven windows of width $0.05,0.1,0.2,0.4,0.8,1.6$, and $3.2 \mathrm{~ms}$ centered at $0.075,0.15,0.3,0.6,1.2,2.4$, and $4.8 \mathrm{~ms}$, respectively. In this paper, I calculated the $B$-field response (Smith and Annan, 2000), but the linearity of the equations in the Appendices are such that the same benefits could also be obtained by measuring $d B / d t$ rather than the $B$-field.
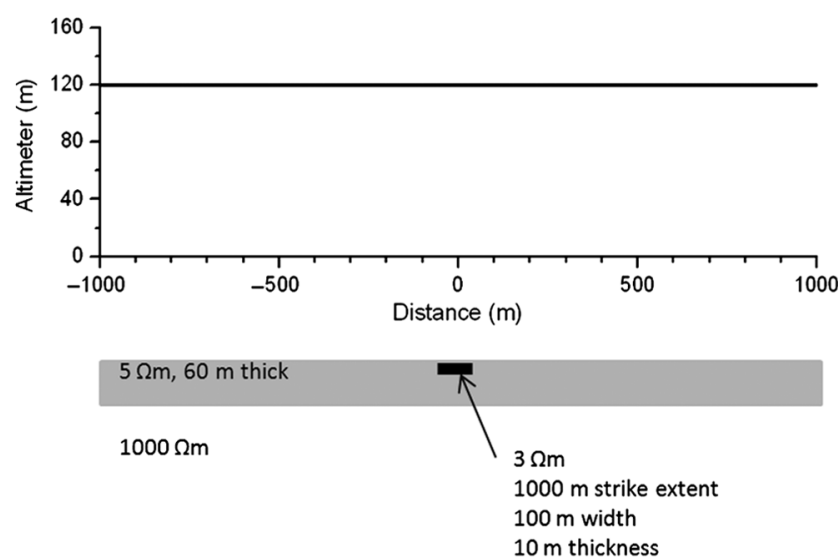

a)

$10 \mathrm{~m}$ thick
Example 1 is a vertical conductor below $60 \mathrm{~m}$ of $5-\Omega \mathrm{m}$ overburden, as shown in Figure 1a, and the $z$ - and $\rho$-component responses are shown in Figure $1 \mathrm{~b}$ on the top and bottom, respectively. The vertical conductor has a strike length of $1000 \mathrm{~m}$, a depth extent of $500 \mathrm{~m}$, a dip of $90^{\circ}$, and a conductance of $20 \mathrm{~S}$. The anomalous response associated with the body is extremely small compared with the large background response associated with the overburden. To identify a response with confidence, an anomalous signal-tonoise ratio greater than one is generally required. In fact, Cheng et al. (2006) suggested a signal-to-noise ratio of three. If the anomalous response is the deviation above the overburden background b)

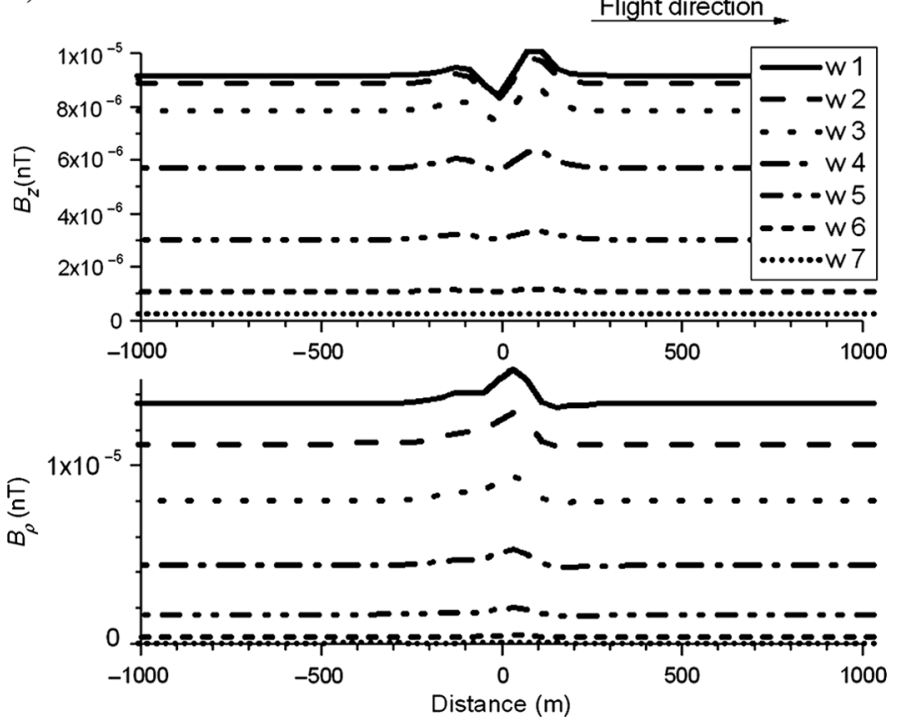

Figure 3. The same as Figure 1, except the anomalous body is a conductive zone near the surface that is modeling a paleochannel. Note that the shape of the anomalous response on the $\rho$-component response at late time is similar to that in Figure 1.

a)

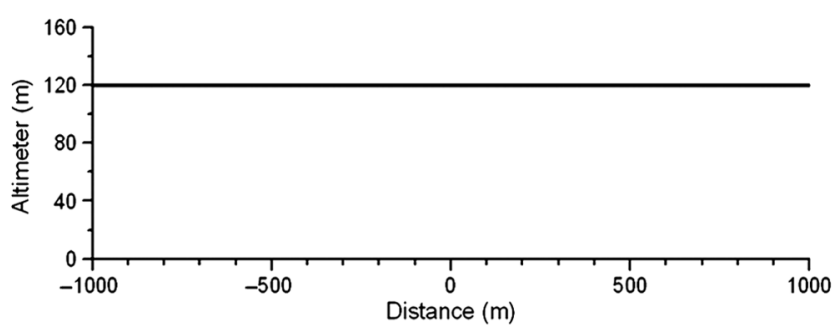

b)

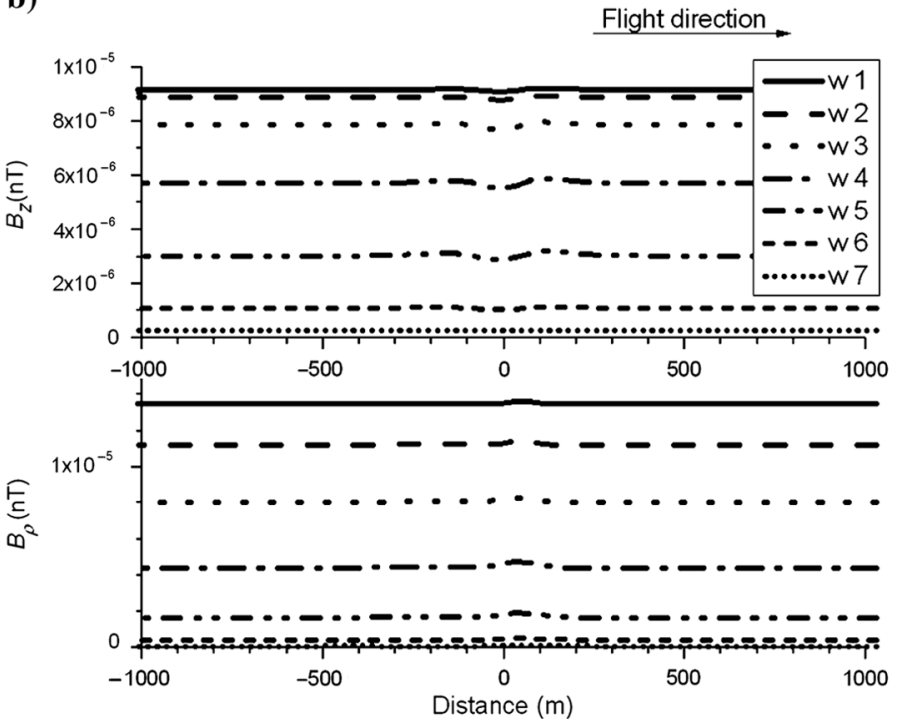

Figure 4. The same as Figure 1, except the anomalous body is a local thickening of the conductive overburden. Note that the shapes of the anomalous responses on the $z$ - and the $\rho$-component responses at all delay times are very similar to those in Figure 1. 
a)

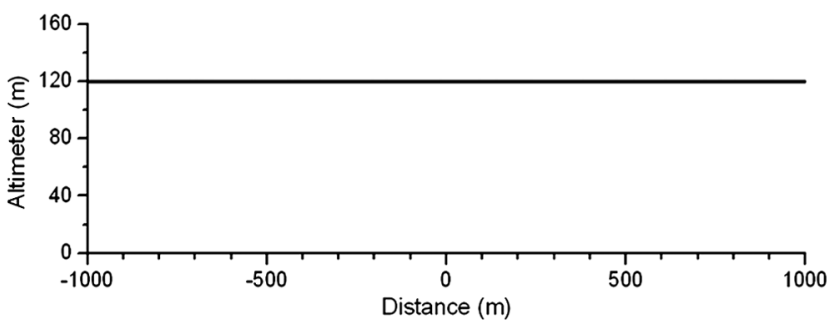

b)

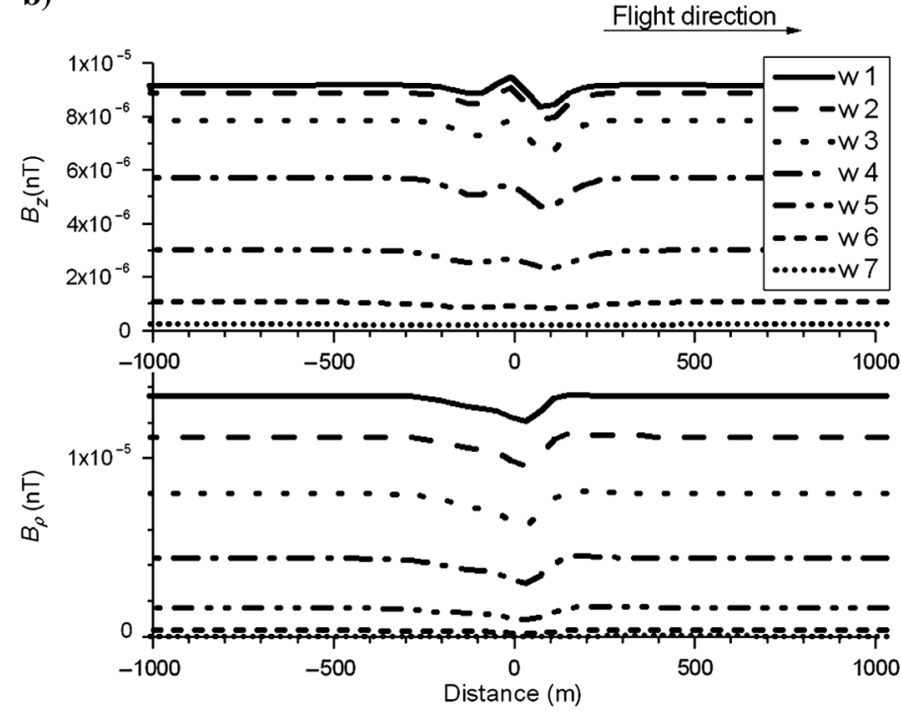

Figure 5. The same as Figure 1, except the anomalous body is a local thinning of the conductive overburden. Note that there are perturbations of the anomalous response that will make identification of the response of buried conductors difficult if they are proximal to the local thinning of the overburden.

a)

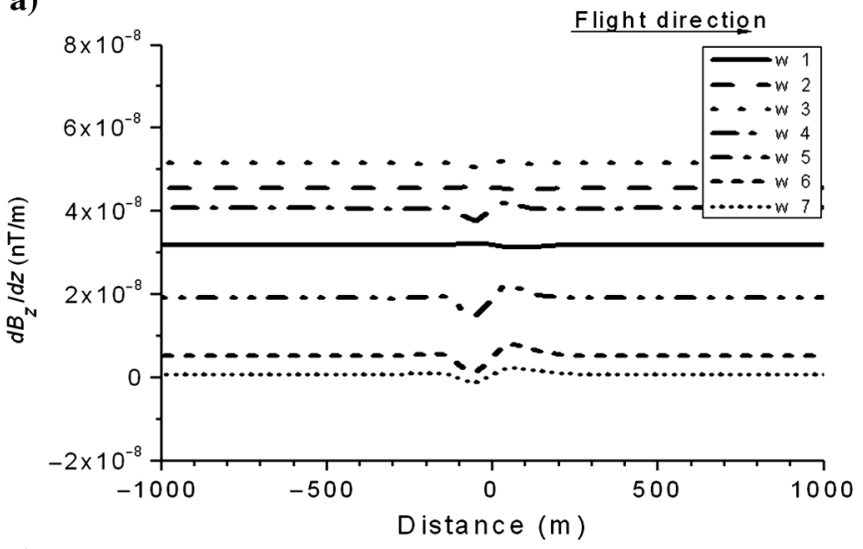

c)

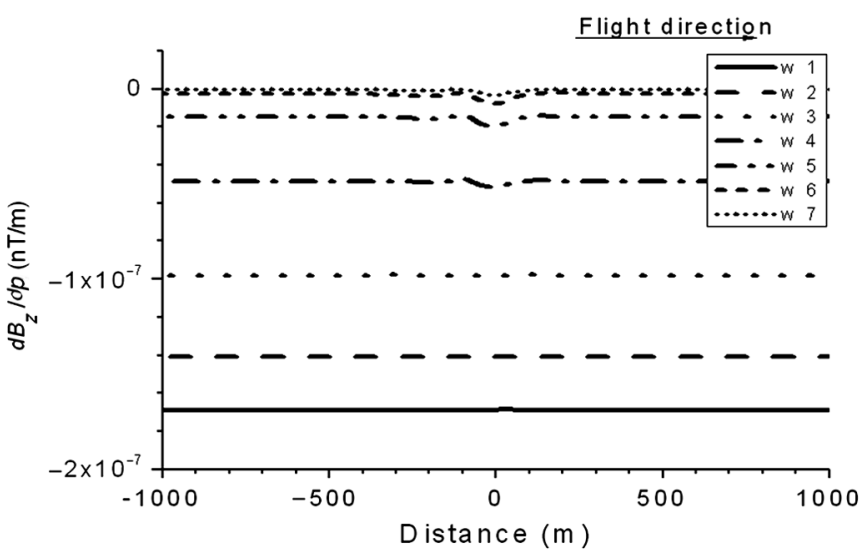

b)

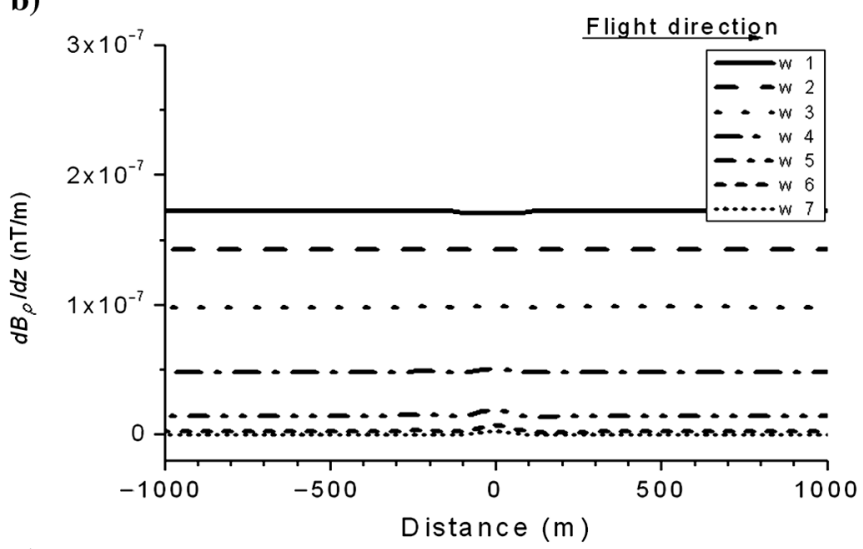

d)

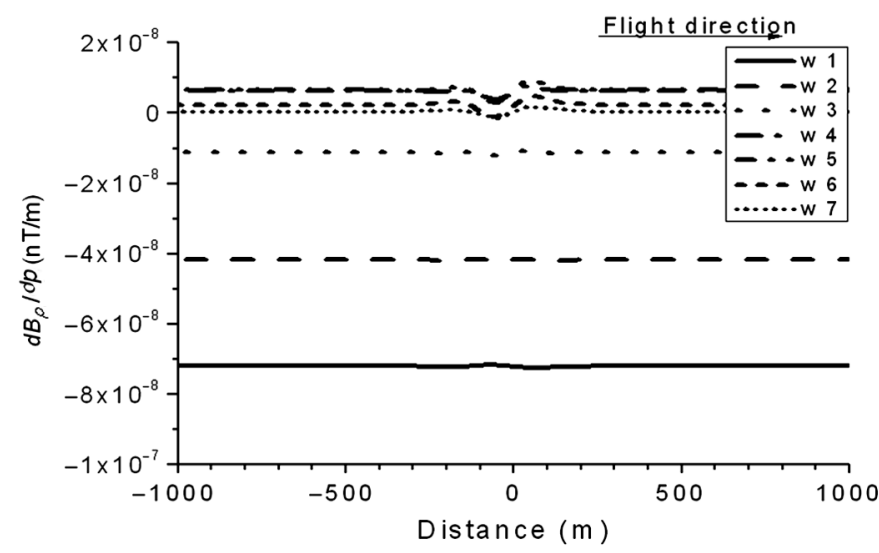

Figure 6. Four different gradients for the vertical conductor model: (a) $d B_{z} / d z$, (b) $d B_{\rho} / d z$, (c) $d B_{z} / d \rho$, and (d) $d B_{\rho} / d \rho$. Note the subtle anomalies on all gradients, with larger anomaly-to-background ratios on the late time and the like gradients $d B_{\rho} / d \rho$ and in particular $d B_{z} / d z$. 
and the noise is the overburden background, then the ratio is much less than one in the early time windows. By the latest window, the ratio is closer to one: for example, on the $z$-component, the latest window has an anomaly-to-background ratio of 0.4 , although the second last and last $\rho$-component windows have ratios of 1.1 and 3.1. In none of these cases would the anomaly be identified and interpreted with confidence. In this example, the signal levels are about $1.0 \times 10^{-7} \mathrm{nT}$. For a real airborne electromagnetic (AEM) system, the intrinsic system noise levels are about 1 pT (Cheng et al., 2006), with dipole moments of about 1,000,000 $\mathrm{Am}^{2}$. For an AEM system with a unit dipole moment, the noise level will be about $10^{-9} \mathrm{nT}$, so in all cases, the measured response is well above the system noise.

The identification of anomalous conductors such as shown in Figure 1 is further complicated by the fact that these anomalies might look similar to the anomalies associated with features or effects that are not of interest. In the second example (Figure 2), I show the response of a uniform overburden when the aircraft altitude deviates by small amounts. The aircraft first moves toward the ground so that the altimeter reading is a low of $116.4 \mathrm{~m}$, then moves away from the ground, so the altimeter reading rises to a high of $124.4 \mathrm{~m}$ and then moves closer a second time to an altimeter low of $117.6 \mathrm{~m}$ (top left panel). This deviation is comparable to that which might occur in normal flying as the pilot strives to obtain a uniform terrain clearance while at the same time avoiding

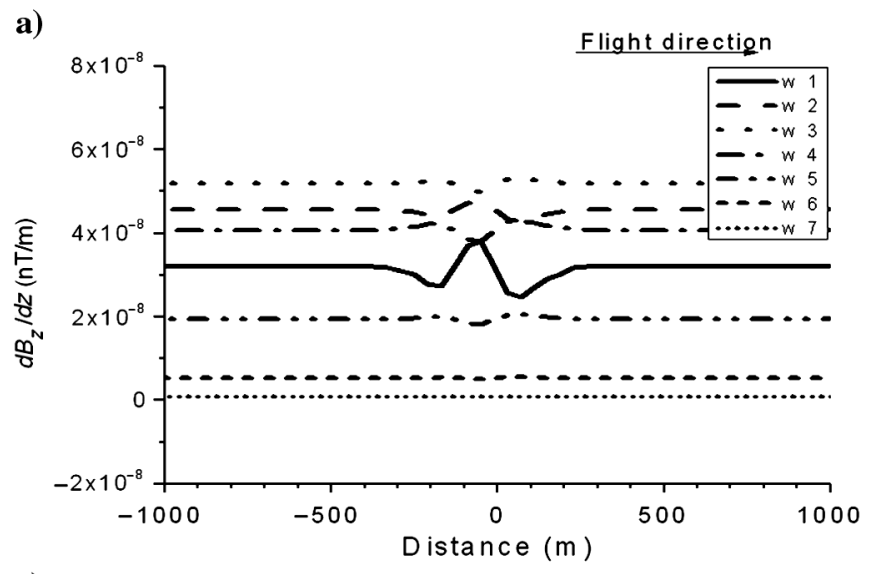

c)

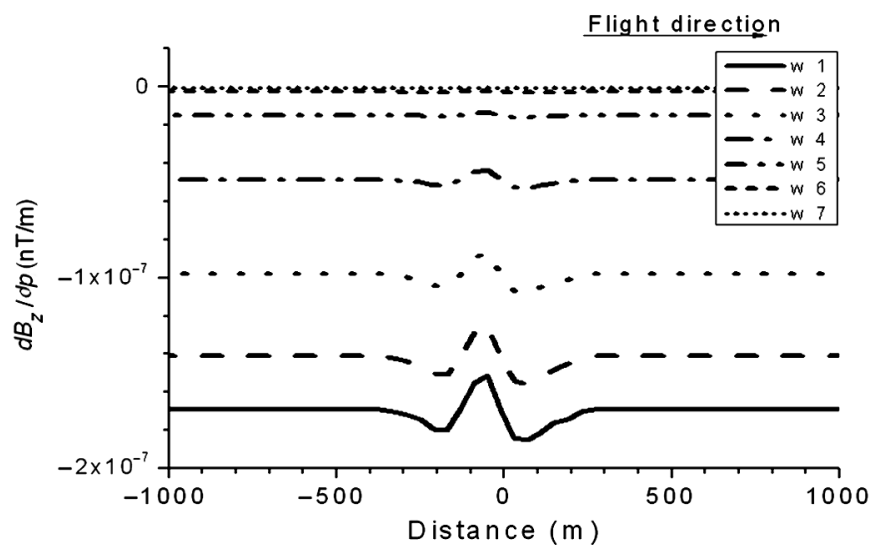

man-made obstacles, trees, or variations in terrain. In this example, the terrain clearance variations were chosen so that the anomaly had a shape that mimicked the $z$-component anomaly of a vertical conductor. However, the $\rho$ - and $z$-components look the same, so if the EM system collects both components, this anomaly might be identified as spurious. If the altimeter variations were at a greater spatial separation on the profile, or the aircraft did not move toward the ground a second time, then this identification of a spurious response would be more problematic.

For completeness, I show the responses associated with models that represent a paleochannel (Figure 3), a local thickening (Figure 4), and a local thinning (Figure 5) of the overburden to show the subtle nature of these types of responses that can distract an interpreter and undermine their confidence to identify zones of interest.

\section{THE MEASUREMENT OF GRADIENTS}

Following the suggestion of Sattel and Macnae (2001), I have calculated four gradient profiles for the vertical component of the magnetic field $\left(B_{z}\right)$ and the horizontal component $\left(B_{\rho}\right)$ measured at the receiver. Once again, EMIGMA software is used to calculate the spatial gradients by differencing the response from two receivers placed $2 \mathrm{~m}$ apart in the $z$ - and $\rho$-directions for calculating the $z$ - and $\rho$-gradients, respectively. The four gradients calculated are $d B_{z} / d z$, $d B_{\rho} / d z, d B_{z} / d \rho$, and $d B_{\rho} / d \rho$. Figure 6 a to $6 \mathrm{~d}$ shows these four

b)

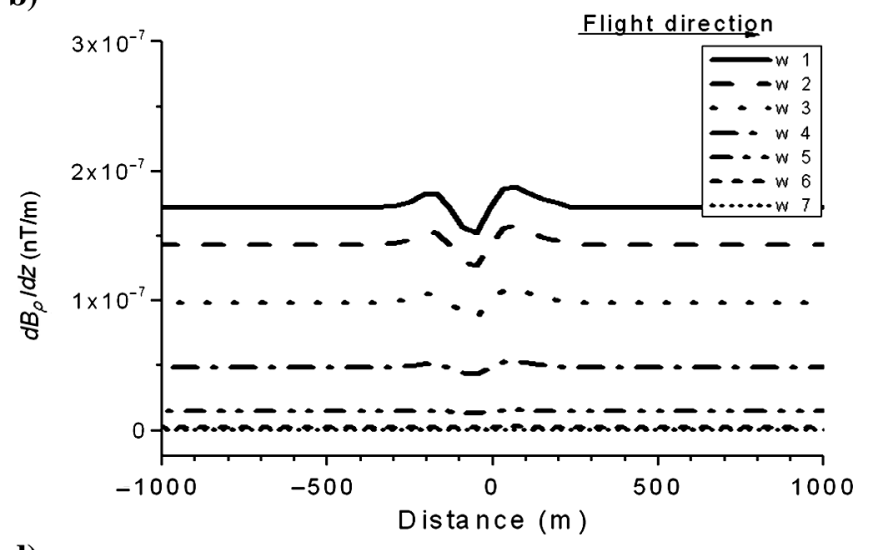

d)

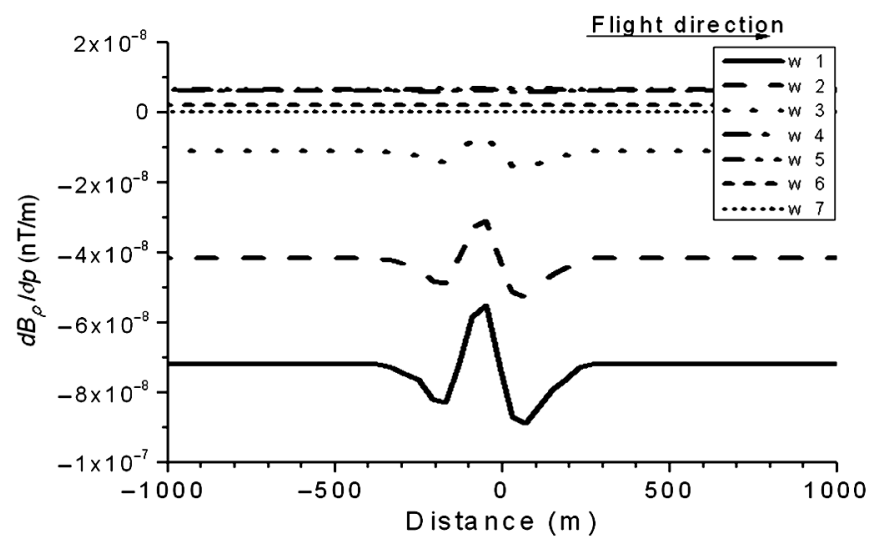

Figure 7. Four different gradients for the variable transmitter altitude model: (a) $d B_{z} / d z$, (b) $d B_{\rho} / d z$, (c) $d B_{z} / d \rho$, and (d) $d B_{\rho} / d \rho$. The anomalies are large at the early time and very small at the late time. 
combinations for each of the seven time windows for the case of the vertical conductor. The size of the anomaly is still small compared to the size of the background, particularly on the two "cross gradients" $d B_{\rho} / d z$ and $d B_{z} / d \rho$; however, the size of the anomaly is slightly larger on the $d B_{\rho} / d \rho$ gradient and larger again on $d B_{z} / d z$. In the latter two "like-gradient" cases, the latetime anomaly-to-background ratio appears larger than in Figures 1 to 5 when the $z$ - and $\rho$-components are measured, so there might be some hope for gradient measurements. In the case in which the altitude is deviating (Figure 7), there are large gradient anomalies on all components, particularly at the early time. The case of the paleochannel (Figure 8) is quite similar, also showing large gradient anomalies on all components, primarily at the early time. If the shape of the variations due to changes in altitude were similar to the gradient anomalies in Figure 8, then distinguishing between these two cases would be difficult. The case of the local thickening of the overburden (Figure 9) shows the best anomaly-to-background ratio in window 4 , at intermediate times; however, the anomaly is still small compared with the background and possibly difficult to identify and discriminate from other anomalies. The final example of gradient profiles, in Figure 10, is for the case when the overburden thins locally. In this case, there are strong responses on all gradients, with the strongest on the like gradients $d B_{\rho} / d \rho$ and $d B_{z} / d z$. From these results, you would conclude that measuring the gradients would enable you to see the strongest responses when

a)

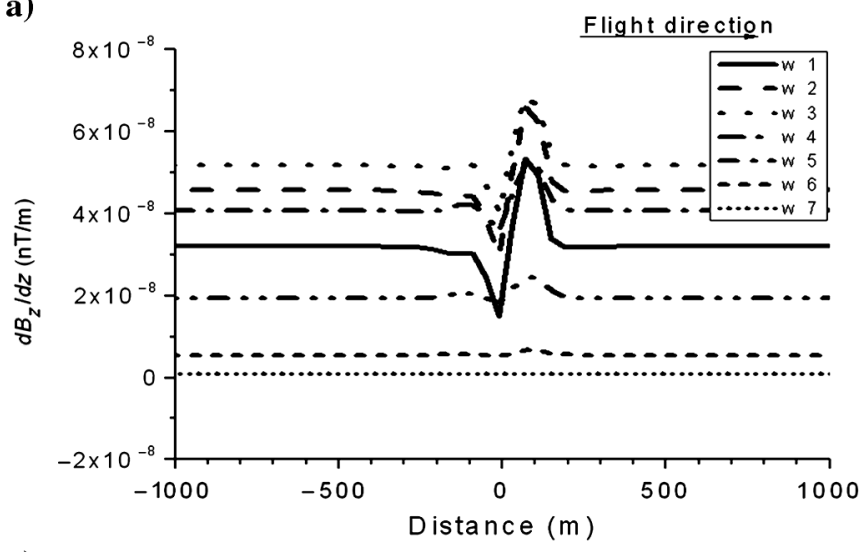

c)

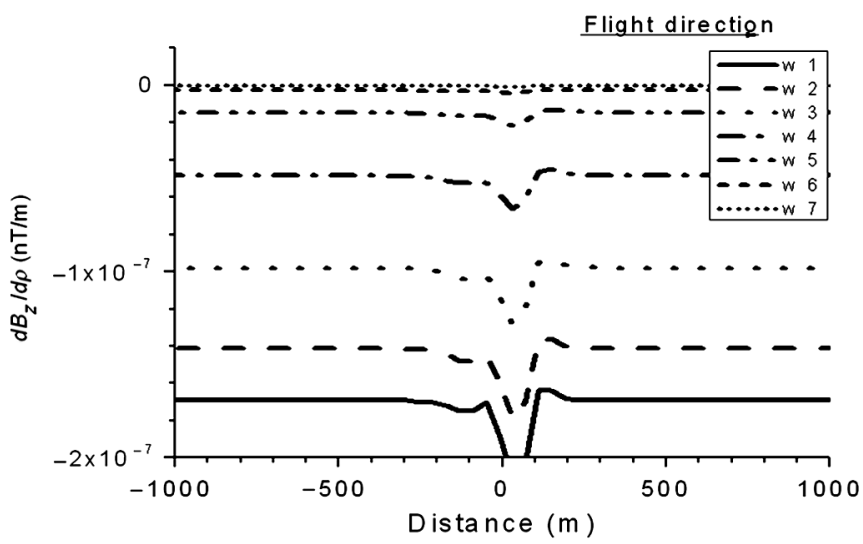

the overburden conductance increases, either through addition of a conductive feature like a paleochannel or when its thickness decreases. The most subtle of all the anomalies is the buried vertical conductor, which is frequently the feature of interest for base metal exploration. Note that these observations are consistent with the conclusions of Sattel and Macnae (2001), who concluded that gradients would give the strongest anomalies for the shallower anomalies.

The dual-loop method was originally proposed for better coupling to vertical conductors and reduction of interference due to distant electromagnetic sources (Spies, 1975). However, the laterally displaced transmitters of opposite sign are essentially a finite difference approximation to a horizontal derivative. In scale model experiments, Spies (1975) finds that the background response due to conductive cover is reduced in the dual-loop system compared with the single-loop system. In his example, the background is a factor of five smaller on the dual-loop configuration and the anomaly-to-background ratio about a factor of seven larger at the late time. Spies acknowledges the logistical difficulties of this configuration, so it is rarely used in the moving-loop mode, but it has recently become more popular in a fixed-loop mode termed InfiniTEM (Boivin, 2007).

In the following, I propose that if gradients can be measured, then a specific combination of the gradients could allow the overburden response to be reduced in a moving loop (e.g., airborne) mode and

b)

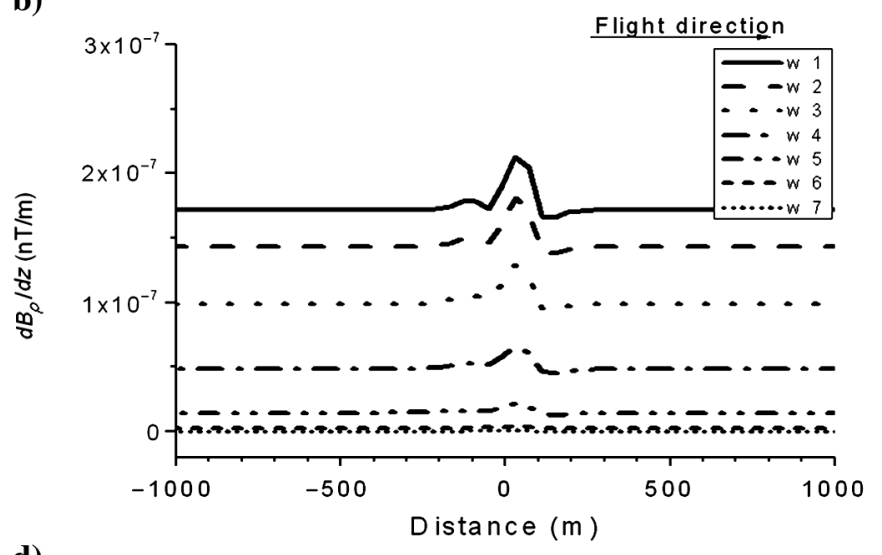

d)

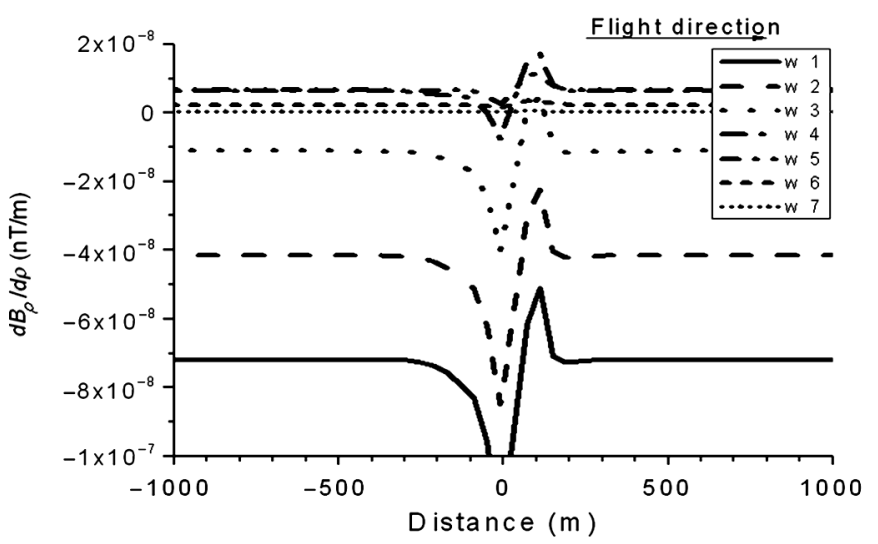

Figure 8. Four different gradients for the paleochannel model: (a) $d B_{z} / d z$, (b) $d B_{\rho} / d z$, (c) $d B_{z} / d \rho$, and (d) $d B_{\rho} / d \rho$. Like the variable altimeter case (Figure 7), the anomalies are large at the early time and smaller at the late time. 
the layered earth or overburden response could be reduced by a factor significantly greater than a factor of five.

\section{COMBINATION OF GRADIENTS}

A careful examination of the plots in Figures 6 to 10 will reveal that the background gradient in the two cross gradients $d B_{\rho} / d z$ and $d B_{z} / d \rho$ are equal in magnitude and opposite in sign. In fact, a theoretical analysis in Appendix A shows that for any half-space or horizontally layered earth excited by a vertical dipole transmitter, these two gradients will always be equal and opposite. In Appen$\operatorname{dix} \mathrm{D}$, this result is generalized for any source and model that is cylindrically symmetric. I have thus defined a quantity $\Delta_{X}$ that is a combination of the cross gradients (equations A-7 and D-3):

$$
\Delta_{X}=\frac{d B_{z}}{d \rho}+\frac{d B_{\rho}}{d z}
$$

which will give a zero response in the background.

Similarly, there is a relation between the like gradient and the horizontal component that allows a second combination $\Delta_{L}$ to be defined that will also be zero in the background regions (equations A-8 and D-6):

$$
\Delta_{L}=\frac{d B_{\rho}}{d \rho}-\frac{d B_{z}}{d z}+\frac{B_{\rho}}{\rho}
$$

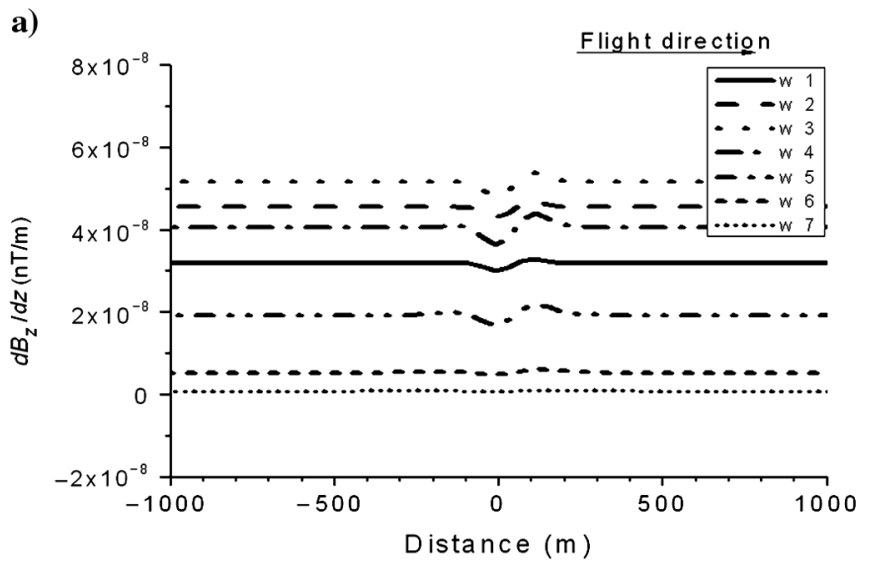

c)

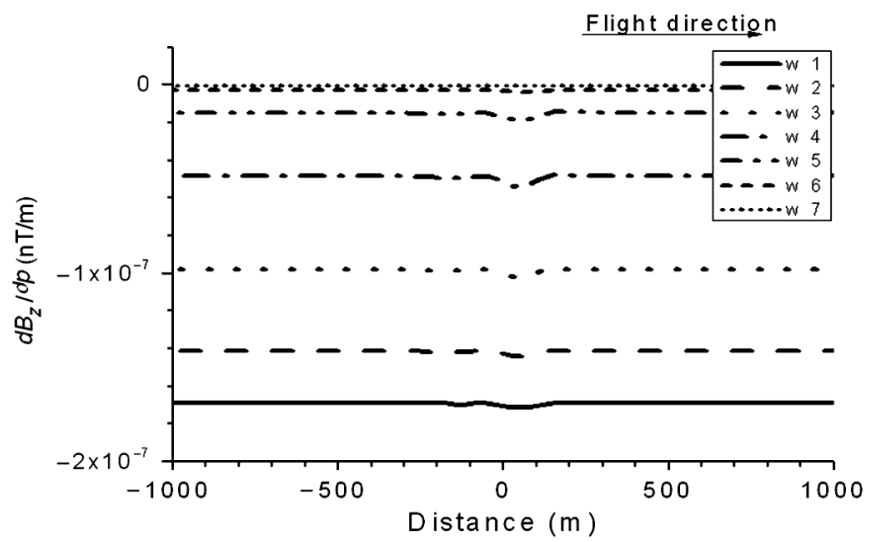

These two combinations have been calculated for the five models considered in this study and plotted in Figures 11 to 15 . In the case of the vertical conductor model (Figure 11), the gradient background has been reduced by two orders of magnitude from about $10^{-7} \mathrm{nT} / \mathrm{m}$ to about $10^{-9} \mathrm{nT} / \mathrm{m}$. In theory, the background should be zero away from the anomalous zone; however, this would require that the modeling algorithm gives the exact response. Because the modeling involves numerical Hankel transforms and a frequency-to-time-domain conversion, an accuracy of about $1 \%$ is considered reasonable; this is consistent with the two-orders-of-magnitude reduction in the background response. In Figure 11, $\Delta_{L}$ and $\Delta_{X}$ show strongly anomalous responses, with anomaly-to-background ratios that are much greater than three, especially on the late delay times. The curves in Figure 11a look unusually sharp, so the response was double checked using both plate algorithms in EMIGMA: the ILN and the VH plate algorithms. The two responses were very similar, and because each algorithm discretizes the plate differently, the unusual response is unlikely to be a discretization issue. As a third check, I calculated the derivatives and then $\Delta_{L}$ and $\Delta_{X}$ using the Win_PLATE program which uses the University of Toronto PLATE algorithm (Annan, 1974). The responses on the $\Delta_{X}$ curves were almost as sharp as in Figure 11a, whereas the curves for $\Delta_{L}$ calculated using PLATE were broader than those in Figure 11a, but much sharper than those in Figure 11b. The difference in the sharpness of the curves on Figure 11a and $11 \mathrm{~b}$ is thus remarkable. My feeling is that the explanation is because the

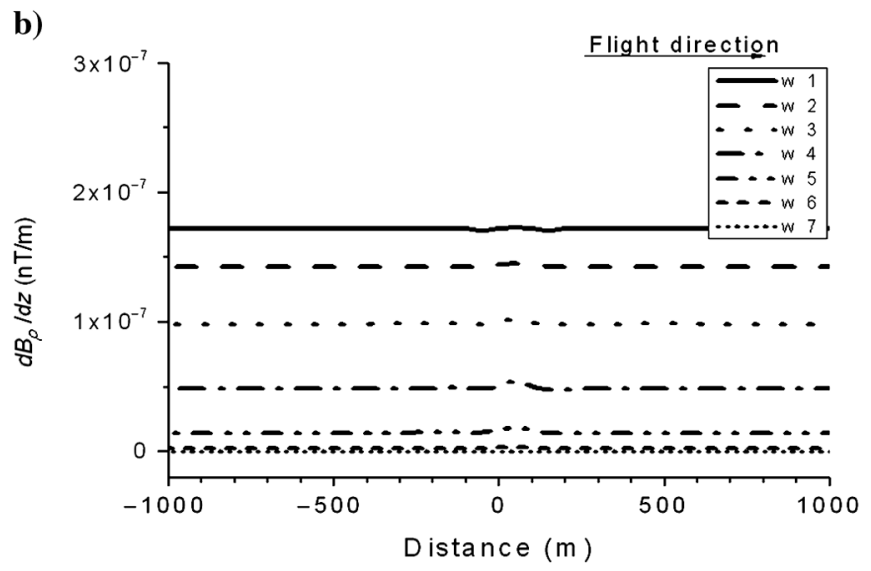

d)

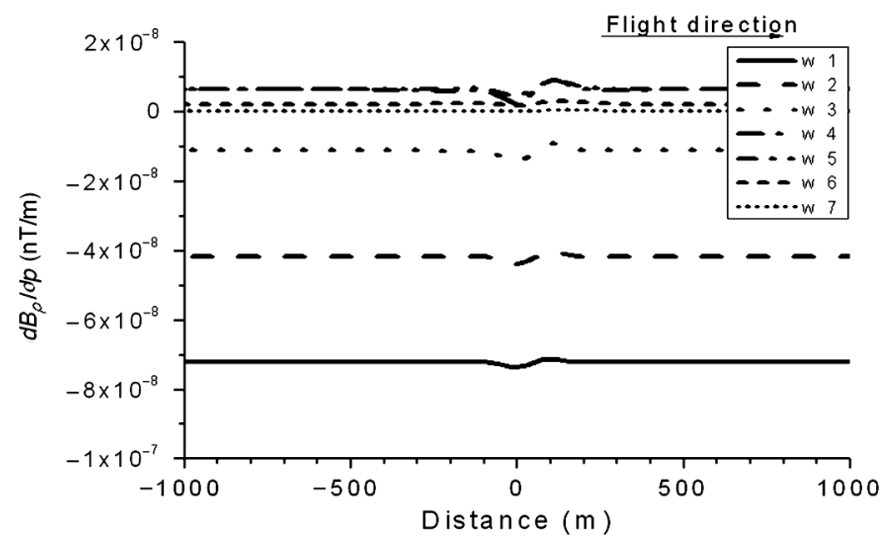

Figure 9. Four different gradients for the model with a local thickening of the overburden: (a) $d B_{z} / d z$, (b) $d B_{\rho} / d z$, (c) $d B_{z} / d \rho$, and (d) $d B_{\rho} / d \rho$. The anomalies are largest in the middle windows and on the like gradients $d B_{\rho} / d \rho$ and in particular $d B_{z} / d z$. 
inductive current flowing in a plate can be approximated by eigencurrents (Annan, 1974). The lowest-order eigencurrent can be approximated by a dipole at distances comparable to the height of an airborne EM receiver, whereas the higher-order eigencurrents would represent quadra-poles and higher-order poles. In Appendix C, I show that for a vertical dipole in free space $\Delta_{X}$ is zero, and using an argument similar to that in Appendix $\mathrm{B}$, it can be shown that $\Delta_{X}$ is also zero for a horizontal dipole in free space. Thus, $\Delta_{X}$ is zero for a dipole of any orientation in free space. At late time, a receiver flying over a dipole in the ground is equivalent to a receiver moving over an arbitrarily oriented dipole in free space. Thus, I expect that $\Delta_{X}$ for the dipole in the ground will be zero, leaving only the $\Delta_{X}$ terms for quadra-poles and higher-order terms. These higher-order terms will have sharper responses, which is what is seen. In Appendix B, I was unable to show that $\Delta_{L}$ was zero for a horizontal dipole, so the dipole term will not cancel, and perhaps this is why broader curves are seen in Figure $11 b$.

For the case of the varying altitude example (Figure 12), there is virtually no signal compared with background on the late-time windows. There is an anomalous response on the early times, but it is generally less than the background. In fact, the only windows that show anomalies are the windows in which the numerical modeling errors are significant. Because these anomalies are only evident in the very early time windows, they would not be considered important when the conductors of interest are conductive buried features that normally have large late-time responses.

a)

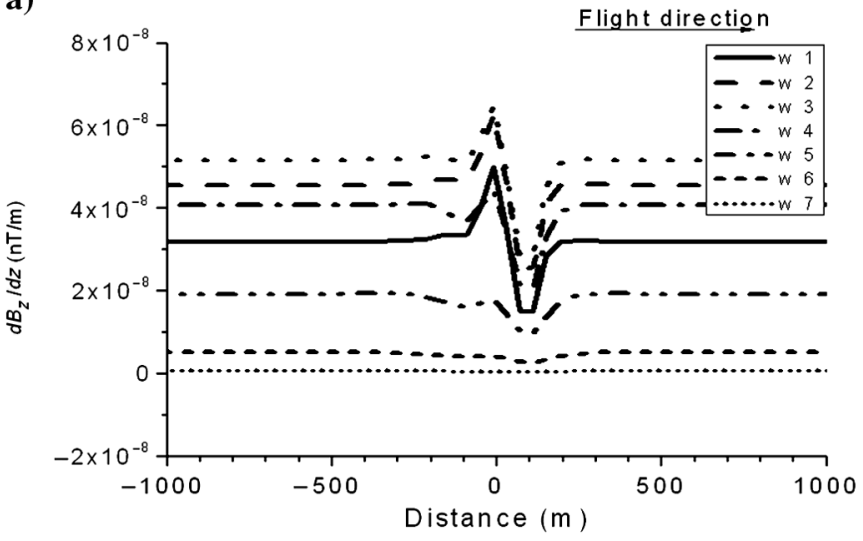

c)

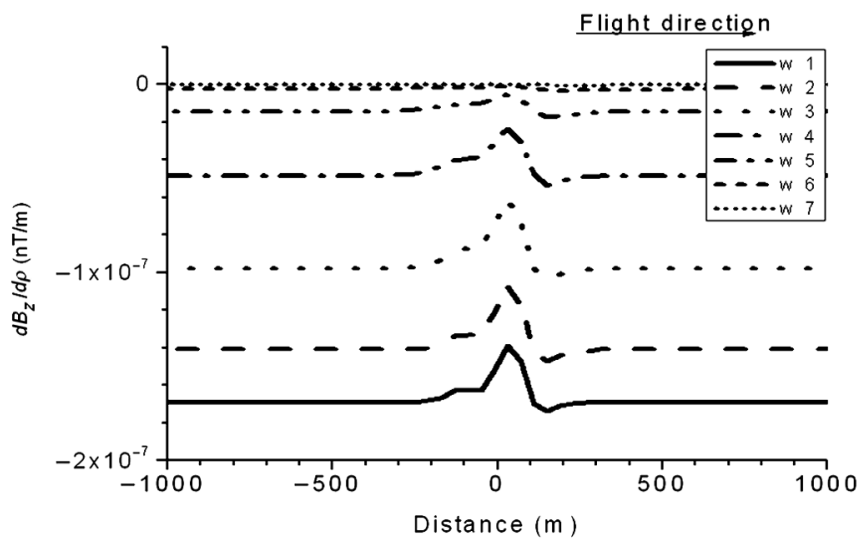

Figure 13 is the conductive near-surface paleochannel model. This shows no strong anomaly on $\Delta_{X}$ in any time windows, but a strong anomaly on $\Delta_{L}$ in most windows, but most particularly in the early time windows. The model representing the local thickening of the overburden (Figure 14) is quite similar, with no strong anomaly on $\Delta_{X}$ in any time windows, but a strong anomaly on $\Delta_{L}$ in most windows. One difference between Figures 13 and 14 is that for the case of Figure 14, the strongest anomalies are in the midtime windows. The model that represents the local thinning of the overburden (Figure 15) also has virtually no anomaly in any window in $\Delta_{X}$, but very strong anomalies in most $\Delta_{L}$ windows. In this case, the strongest anomalies appear to be at the earliest time.

The combination $\Delta_{X}$ has a strong anomaly at all times, and in particular late times, over the conductor of interest (the vertical conductor). The other models all have weak $\Delta_{X}$ anomalies over this type of body; however, they do have strong responses in $\Delta_{L}$. If the strongest $\Delta_{L}$ anomalies are in the early windows, then the feature is likely to be shallow; if the anomalies are strongest at the later time, then the feature is likely deeper.

\section{DISCUSSION}

The dipole excitation field assumed in Appendix A means that the size of the transmitter must effectively be small compared with the offset of the transmitter and receiver. For fixed-wing AEM systems, the loop size is much smaller than the transmitter-receiver

b)

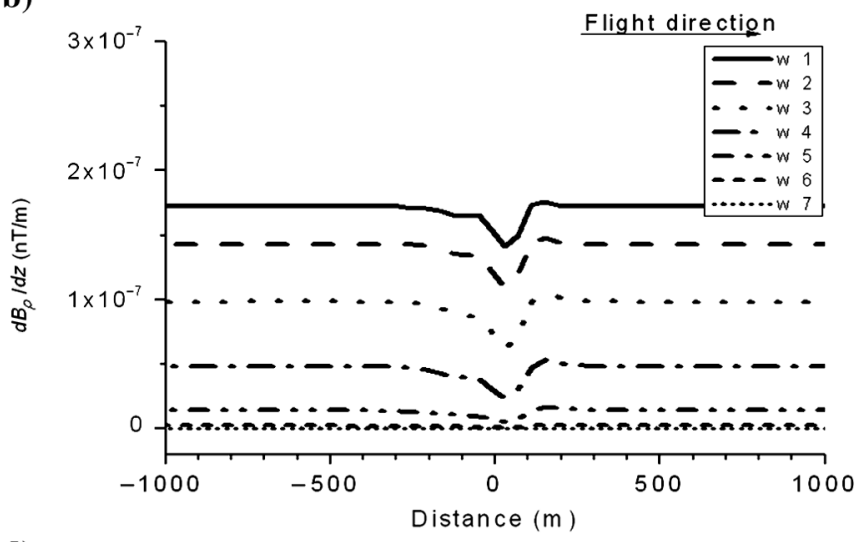

d)

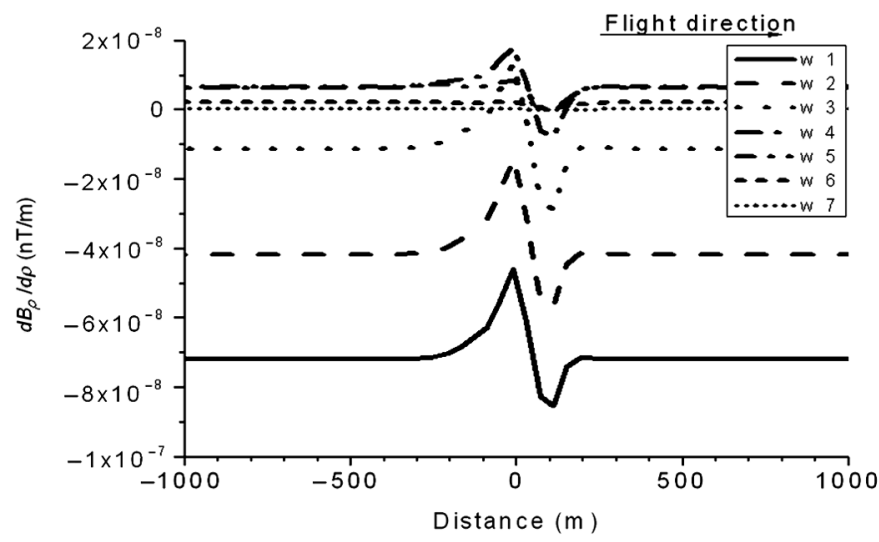

Figure 10. Four different gradients for the model with a local thinning of the overburden: (a) $d B_{z} / d z$, (b) $d B_{\rho} / d z$, (c) $d B_{z} / d \rho$, and (d) $d B_{\rho} / d \rho$. The anomalies are largest in the early windows and on the like gradients $d B_{\rho} / d \rho$ and in particular $d B_{z} / d z$. 
separations; however, for the recently developed time-domain helicopter systems (Allard, 2007; Smith et al., 2009) the loop is large compared with the transmitter-receiver offset, so the dipole transmitter assumption is not as valid.

The theoretical developments in Appendices A and B are for the secondary fields above a conductive layered half-space. Very similar relations apply for the fields from a dipole in free space, with a reversal in the sign of the $z$-derivative term because the source is above the receiver, not below the receiver. Hence, calculating the combinations in equations $\mathrm{C}-1$ and $\mathrm{C}-2$ for the gradients of the in-phase primary fields from an airborne EM system should give zero unless there is an in-phase secondary coming from conductive bodies below the ground surface.

The examples in this paper are for airborne systems, but the approach will work for ground EM systems provided that the separation is large compared to the transmitter loop size.

One difficulty that must be addressed when measuring the gradients is dealing with rotation of the airborne receiver coils, in which case the receivers are not measuring the true vertical and horizontal components. Inertial measurement units are now becoming

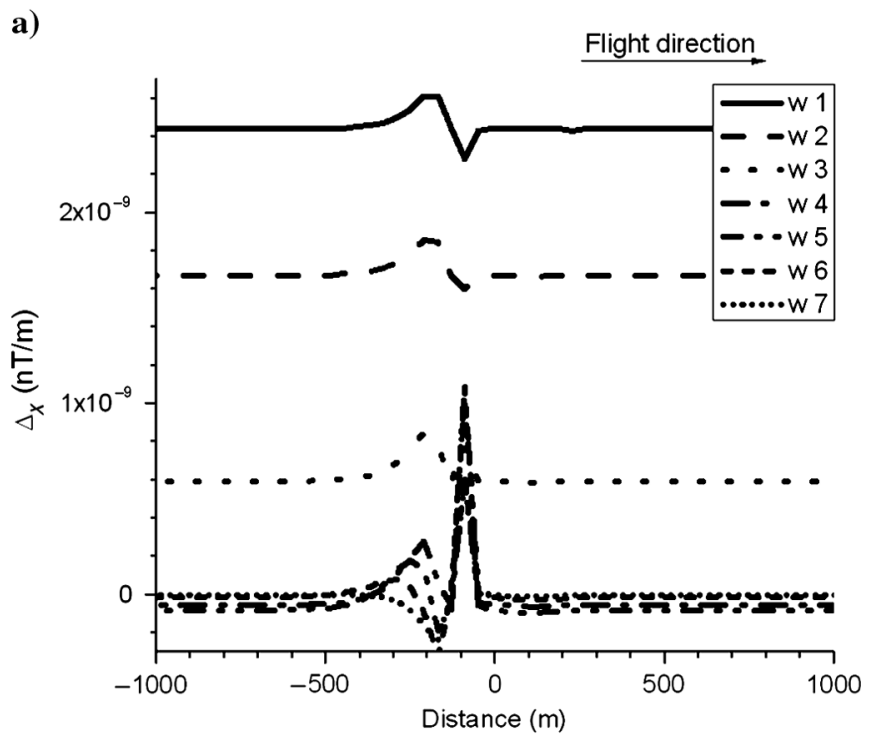

b)

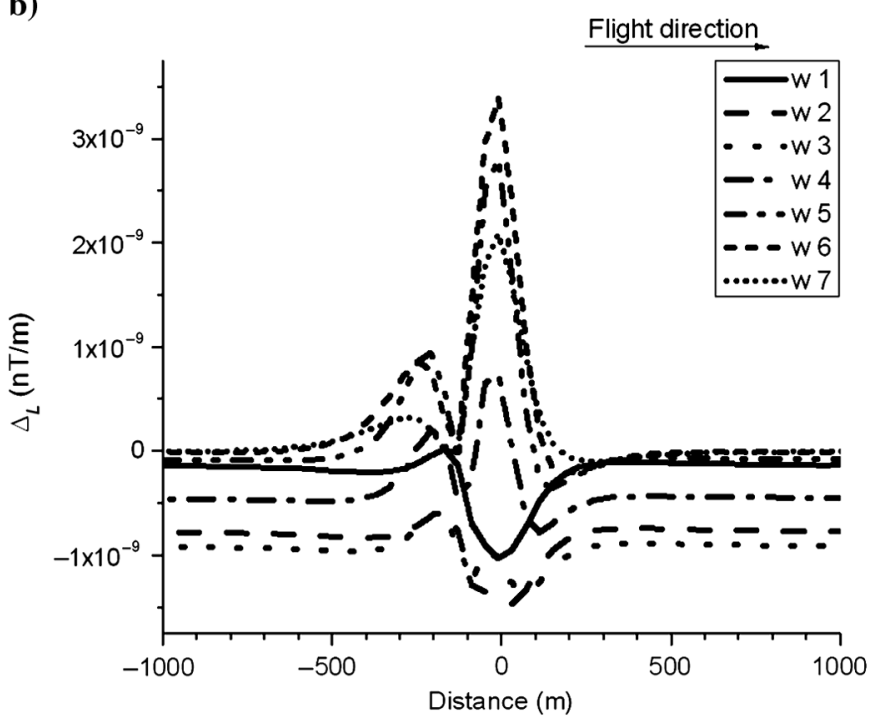

Figure 11. Two combinations of gradients involving (a) cross-gradient terms and (b) like-gradient terms. In this case, the model is the vertical conductor. The background has been reduced to close to zero, and the anomaly-to-background ratio is large on the cross- and like-gradient combinations.

a)

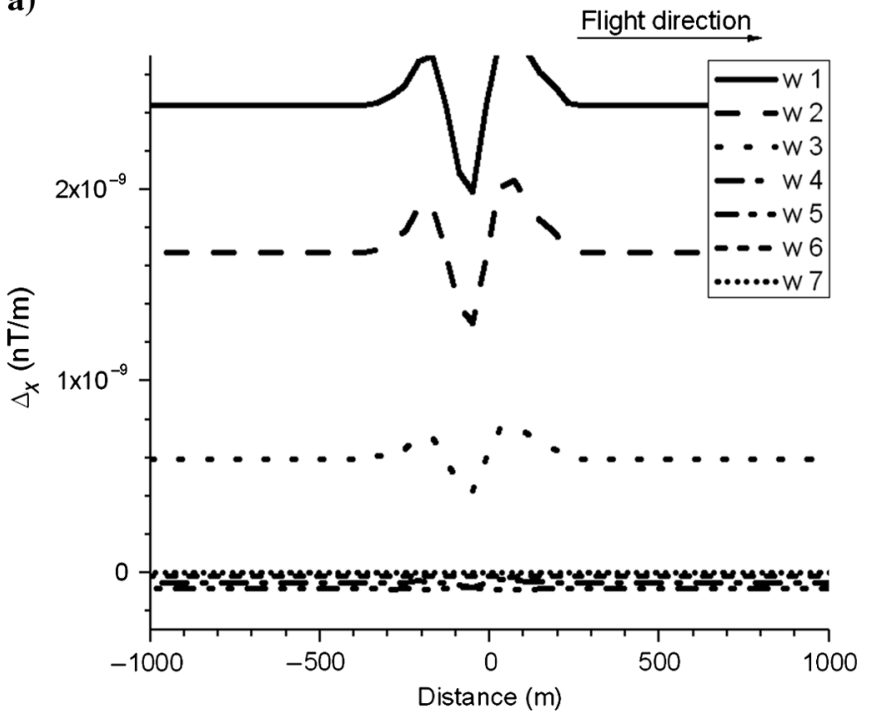

b)

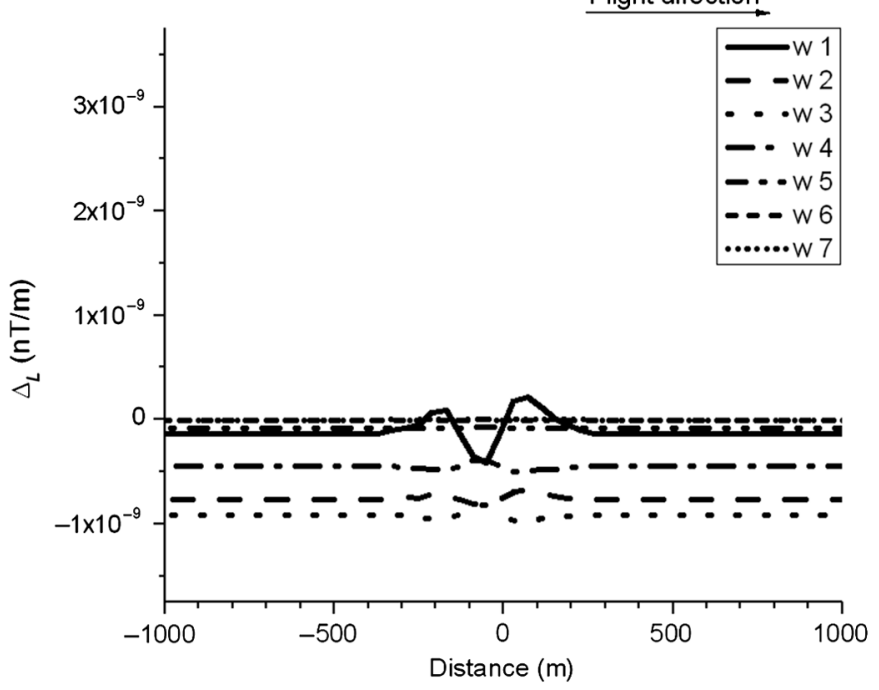

Figure 12. Two combinations of gradients involving (a) cross-gradient terms and (b) like-gradient terms. In this case, the model is the uniform conductor with the transmitter altitude varying. The background has been reduced to close to zero, and there are only anomalies at the very early time when there is a slight inaccuracy of the modeling algorithm so that the background is not identically zero. 
available and these can be used to monitor orientation changes and correct for them. Another way of compensating for orientation changes is to use the primary fields (Smith and Annan, 1997).

A second difficulty is that the airborne transmitter is not a dipole that is always vertical, because the aircraft changes pitch. In Appendix B, I show that for a horizontal dipole oriented in the flight direction, the combination $\Delta_{X}$ is still zero. This means that a transmitter that is a linear combination of vertical and horizontal dipoles (i.e., pitching) will also have a zero $\Delta_{X}$.

There are a number of approaches for building a receiver system for measuring the gradients: placing the multiple sensors on a rigid structure (such a structure would in practice have to be small) or having the sensors a larger variable distance apart and monitoring the changes in the distance and orientation. The most appropriate choice would be the approach that gives the lower noise levels, and this is best determined by building a system and measuring the noise levels, a task beyond the scope of this paper and beyond the research budget of the author.

The fact that the anomalous response on $\Delta_{X}$ seen in Figure 11a is significantly sharper than the response in Figure $11 \mathrm{~b}$ could be an advantage because $\Delta_{X}$ could provide greater resolution than the other combination. One potential drawback is that $\Delta_{X}$ is zero if the a)

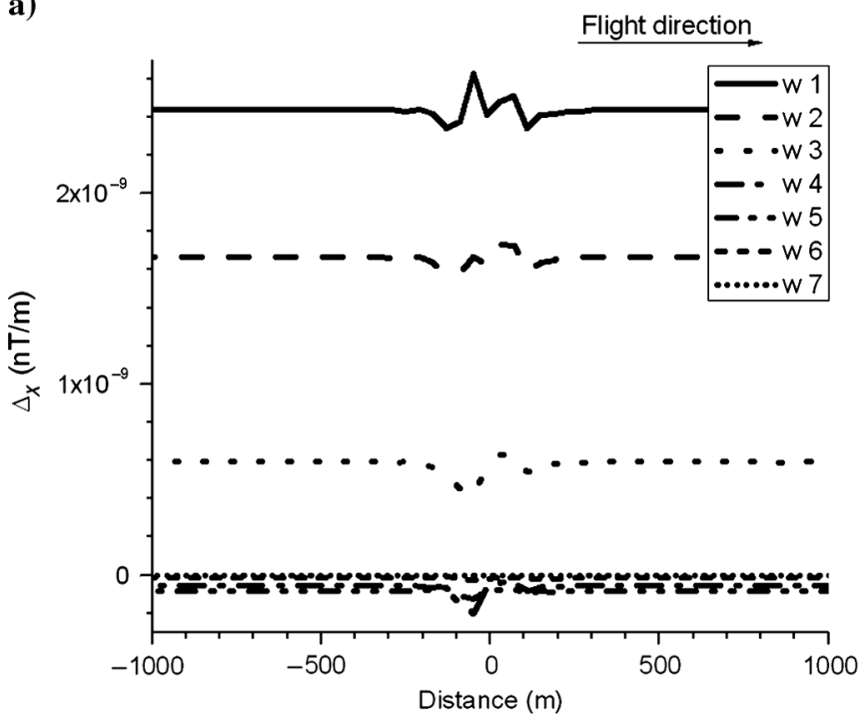

b)

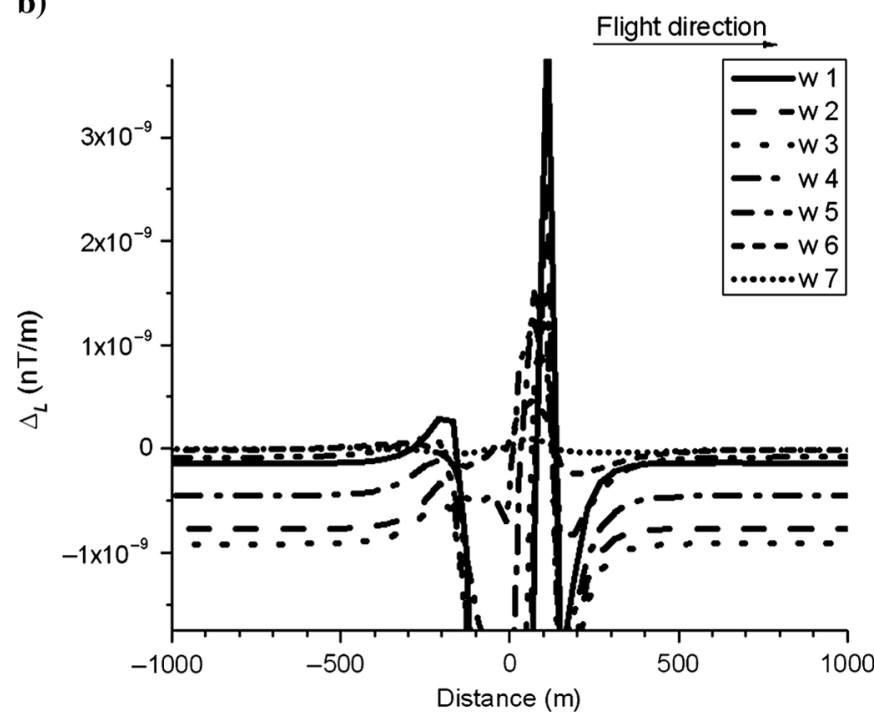

Figure 13. Two combinations of gradients involving (a) cross-gradient terms and (b) like-gradient terms. In this case, the model is the paleochannel. The background has been reduced to close to zero, and the anomaly-to-background ratio is small on the cross-gradient combination and larger on the like-gradient combination.

a)

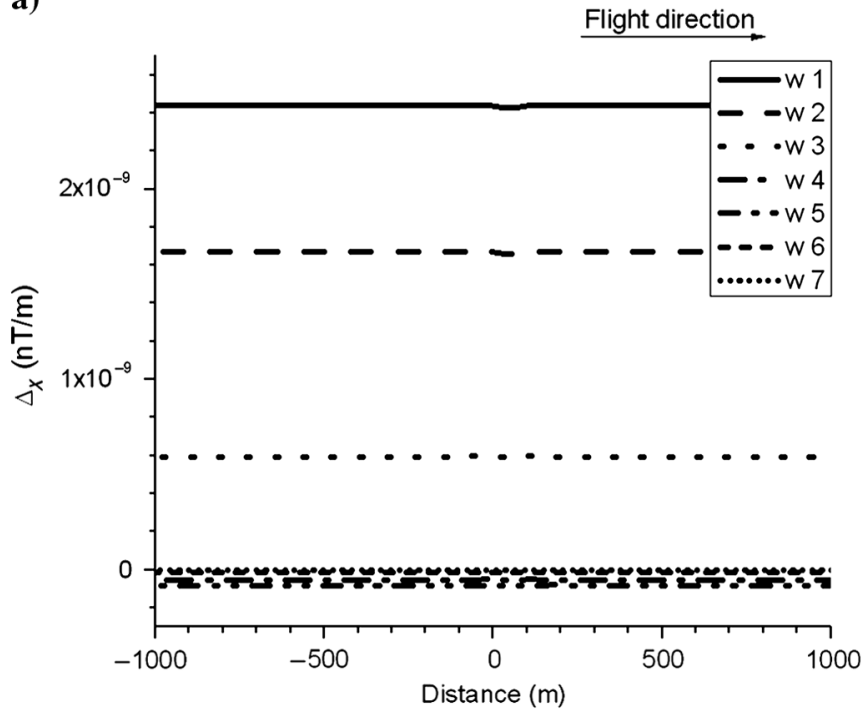

b)

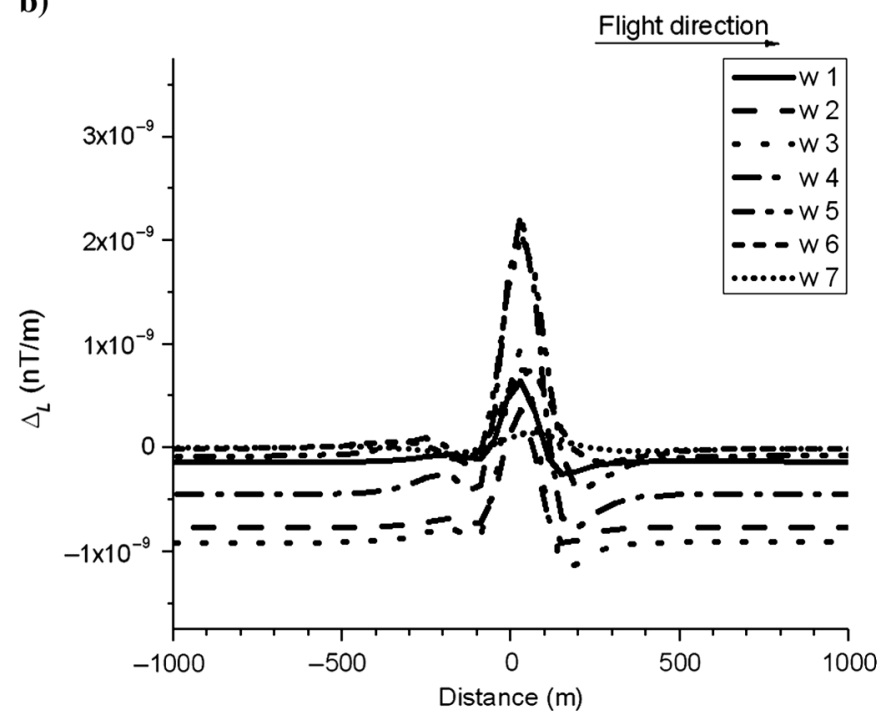

Figure 14. Two combinations of gradients involving (a) cross-gradient terms and (b) like-gradient terms. In this case, the model is the local thickening of the overburden. The background has been reduced to close to zero, and the anomaly-to-background ratio is small on the crossgradient combination and larger on the like-gradient combination (but not as large as the previous case). 
a)

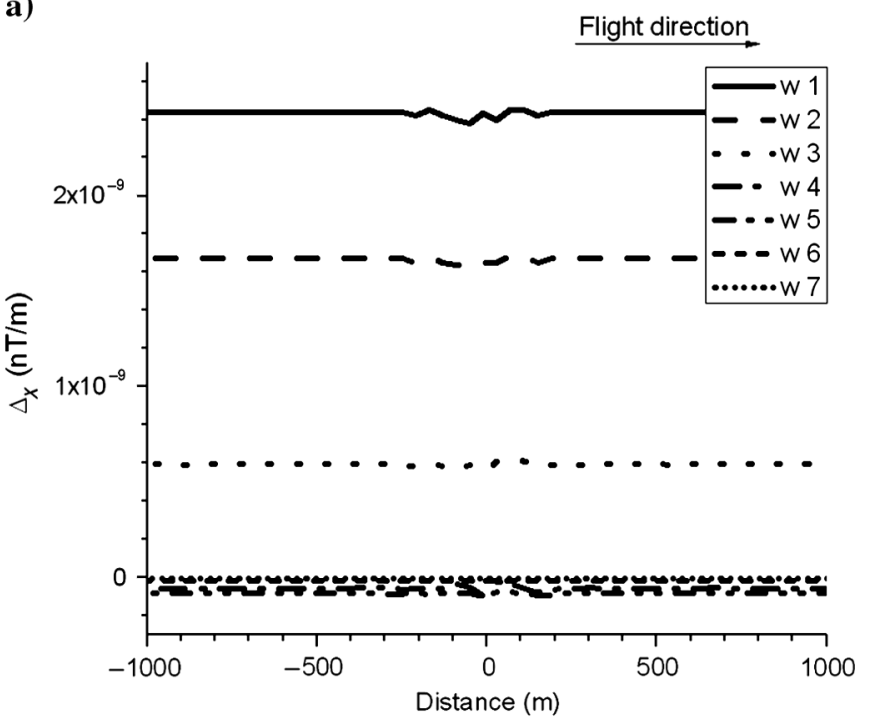

b)

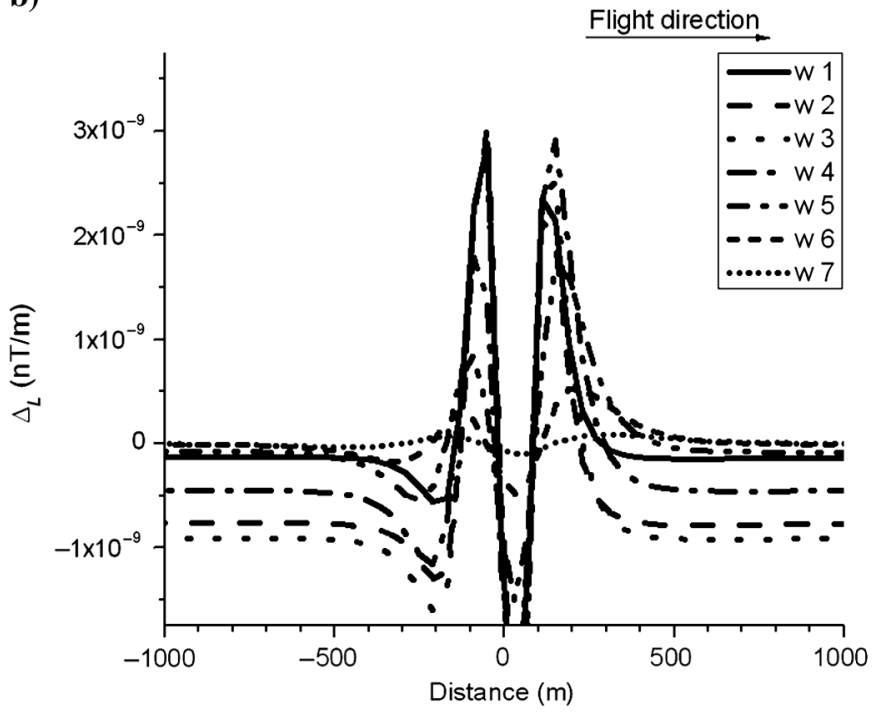

Figure 15. Two combinations of gradients involving (a) cross-gradient terms and (b) like-gradient terms. In this case, the model is the local thinning of the overburden. The background has been reduced to close to zero, and the anomaly-to-background ratio is small on the crossgradient combination and larger on the like-gradient combination (but larger than the previous case and comparable in shape and magnitude but opposite in sign to what is seen in Figure 13).

conductor in the ground is a pure dipole (Appendix B). Fortunately, the conductor modeled (a vertical plate) has higher-order modes, so the response is nonzero. Most real conductors are expected to be more complicated than a simple dipole.

\section{CONCLUSIONS}

It is difficult to identify the response of a buried vertical conductor when there is conductive material overlying the conductor. The anomalous response of the conductor can be subtle compared to the background response and can be similar to the response of other conductive features or geometric effects such as aircraft altimeter variations, a near-surface paleochannel, and a local thinning or a local thickening of the overburden.

Measuring the gradients of the buried conductor can in some cases provide a slight improvement in the anomaly-to-background ratio, particularly on the $d B_{z} / d z$ gradient. However, the anomalyto-background ratio is even larger for all the other models, so identification of buried conductors will be difficult if there are local changes in the thickness of the overburden or changes in its conductance (e.g., due to paleochannels).

In theory, two specific combinations of gradients and fields that involve cross gradients and like gradients $\Delta_{X}$ and $\Delta_{L}$ should be zero over a horizontally layered conductive earth due to the cylindrical symmetry of the earth. Calculating these combinations shows that the vertical conductor has a significant anomaly-to-background ratio on these combinations in all time windows, but most particularly at late times. The other variable overburden models all have weak anomaly-to-background ratios on the cross-component combination and larger anomalies on the like-component combination. The relative delay time of the largest anomaly on the like-gradient combination (early or late) can give an indication of whether the anomalous feature is shallow or deep, respectively.

Hence the gradient combination $\Delta_{X}$ and $\Delta_{L}$ could be used to identify conductors buried below conductive overburden if a system capable of measuring gradients can be built.

\section{ACKNOWLEDGMENTS}

I would like to thank Peter Annan for feedback and suggesting a number of points that require clarification and for prompting the alternate derivations in Appendix D. Aspects of this paper are in a patent application belonging to Fugro Airborne Surveys.

\section{APPENDIX A}

\section{VERTICAL DIPOLE TRANSMITTER OVER A LAYERED HALF-SPACE}

The $z$ and $\rho$ components of the secondary magnetic field response $H$ of a layered earth when excited by a vertical dipole transmitter are given in Ward and Hohmann (1988):

$$
H_{\rho}=\frac{m}{4 \pi} \int_{0}^{\infty} r_{\mathrm{TE}} e^{\lambda(z-h)} \lambda^{2} J_{1}(\lambda \rho) \mathrm{d} \lambda,
$$

and

$$
H_{z}=\frac{m}{4 \pi} \int_{0}^{\infty} r_{\mathrm{TE}} e^{\lambda(z-h)} \lambda^{2} J_{0}(\lambda \rho) \mathrm{d} \lambda,
$$

where $m$ is the dipole moment of the transmitter, $r_{\mathrm{TE}}$ is the reflection coefficient for a horizontally layered earth, $\lambda$ is the wavenumber, $\rho$ is the horizontal offset of the receiver from the transmitter, and $J_{1}$ is the Bessel function order $l$. Because I follow Ward and Hohmann (1988), $z$ is positive downward with $z=0$ being the ground surface, so the receiver being above the ground will have a negative $z$ value, but the $h$ is the height of the transmitter and $h$ is positive above the ground.

Taking the derivative with respect to $\rho$ of equation 1 gives

$$
\frac{d H_{\rho}}{d \rho}=\frac{m}{4 \pi} \int_{0}^{\infty} r_{\mathrm{TE}} e^{\lambda(z-h)} \lambda^{2} \frac{d J_{1}(\lambda \rho)}{d x} \mathrm{~d} \lambda .
$$


The derivative of the first-order Bessel function can be evaluated using equation 9.1.30 of Abramowitz and Stegun (1965):

$$
\frac{d J_{1}(\lambda \rho)}{d \rho}=\lambda J_{0}(\lambda \rho)-J_{1}(\lambda \rho) / \rho,
$$

which when substituted in equation A-3 can be shown to give

$$
\frac{d H_{\rho}}{d \rho}=\frac{d H_{z}}{d z}-\frac{H_{\rho}}{\rho}
$$

because the additional $\lambda$ in the integral can be generated by taking a derivative with respect to $z$.

Similarly, taking the $\rho$ derivative of equation A-2 and using equation 9.1.28 of Abramowitz and Stegun (1965) gives

$$
\frac{d H_{z}}{d \rho}=-\frac{d H_{\rho}}{d z}
$$

Because $B=\mu H$, I can define a combination $\Delta_{L}$ that involves like derivatives and components,

$$
\Delta_{L}=\frac{d B_{\rho}}{d \rho}-\frac{d B_{z}}{d z}+\frac{B_{\rho}}{\rho}
$$

and another combination $\Delta_{X}$ with cross derivatives and components,

$$
\Delta_{X}=\frac{d B_{z}}{d \rho}+\frac{d B_{\rho}}{d z}
$$

Using equations A-5 and A-6, both of these combinations should be zero for a layered conductive half-space.

\section{APPENDIX B}

\section{HORIZONTAL DIPOLE TRANSMITTER OVER A LAYERED HALF-SPACE}

The $z$-and $\rho$-components of the secondary magnetic field response $H$ of a layered earth when excited by a horizontal dipole $x$-directed transmitter are given in Ward and Hohmann (1988):

$$
\begin{aligned}
H_{\rho}= & \frac{m}{4 \pi}\left(\frac{1}{\rho}-\frac{2 x^{2}}{\rho^{3}}\right) \int_{0}^{\infty} r_{\mathrm{TE}} e^{\lambda(z-h)} \lambda J_{1}(\lambda \rho) \mathrm{d} \lambda \\
& +\frac{m}{4 \pi} \frac{x^{2}}{\rho^{2}} \int_{0}^{\infty} r_{\mathrm{TE}} e^{\lambda(z-h)} \lambda^{2} J_{0}(\lambda \rho) \mathrm{d} \lambda,
\end{aligned}
$$

and

$$
H_{z}=\frac{m}{4 \pi} \frac{x}{\rho} \int_{0}^{\infty} r_{\mathrm{TE}} e^{\lambda(z-h)} \lambda^{2} J_{1}(\lambda \rho) \mathrm{d} \lambda
$$

where the symbols are as before, but the dipole is oriented along the $x$-axis and $\rho=\sqrt{ }\left(x^{2}+y^{2}\right)$. If we assume that the receiver is below the dipole axis, then $y=0$ and $\rho=x$, giving

$$
\begin{aligned}
H_{\rho}= & -\frac{m}{4 \pi} \frac{1}{\rho} \int_{0}^{\infty} r_{\mathrm{TE}} e^{\lambda(z-h)} \lambda J_{1}(\lambda \rho) \mathrm{d} \lambda \\
& +\frac{m}{4 \pi} \int_{0}^{\infty} r_{\mathrm{TE}} e^{\lambda(z-h)} \lambda^{2} J_{0}(\lambda \rho) \mathrm{d} \lambda,
\end{aligned}
$$

and

$$
H_{z}=\frac{m}{4 \pi} \int_{0}^{\infty} r_{\mathrm{TE}} e^{\lambda(z-h)} \lambda^{2} J_{1}(\lambda \rho) \mathrm{d} \lambda
$$

Taking the derivative with respect to $\rho$ of equation B-4 gives

$$
\frac{d H_{z}}{d \rho}=\frac{m}{4 \pi} \int_{0}^{\infty} r_{\mathrm{TE}} e^{\lambda(z-h)} \lambda^{2} \frac{d J_{1}(\lambda \rho)}{d p} \mathrm{~d} \lambda .
$$

Using equation A-4 gives

$$
\frac{d H_{z}}{d \rho}=\frac{m}{4 \pi} \int_{0}^{\infty} r_{\mathrm{TE}} e^{\lambda(z-h)} \lambda^{2}\left(\lambda J_{0}(\lambda \rho)-J_{1}(\lambda \rho) / \rho\right) \mathrm{d} \lambda
$$

Comparing this with equation B-3, we can write

$$
\frac{d H_{z}}{d \rho}=-\frac{d H_{\rho}}{d z},
$$

which is the same as equation A-6. Hence, like the vertical dipole case, $\Delta_{X}$ will be zero for an $x$ directed dipole.

Unfortunately, I have not been able to establish a relation for the like terms in the horizontal dipole case.

For the case of a $y$-directed dipole transmitter, the vertical and horizontal $(x)$ derivatives of the radial and vertical fields are zero on the $x$-axis. Hence it appears that the transmitter roll should have a minimal impact.

\section{APPENDIX C}

\section{DIPOLE FIELDS IN FREE SPACE}

For the case of a dipole source in free space, the equations for the field are given by equations A-1, A-2, B-1, and B-2, except $r_{\mathrm{TE}} e^{\lambda(z-h)}$ is replaced by $e^{-\lambda(z-h)}$. The negative sign in the exponential means that we must redefine our two combinations. The combination $\Delta_{X}$ with cross derivatives becomes

$$
\Delta_{X}=-\frac{d B_{z}}{d \rho}+\frac{d B_{\rho}}{d z},
$$

and this will be zero for a vertical and horizontal dipole. The combination $\Delta_{L}$ that involves like derivatives and components becomes

$$
\Delta_{L}=\frac{d B_{\rho}}{d \rho}+\frac{d B_{z}}{d z}+\frac{B_{\rho}}{\rho}
$$

and this will be zero for the vertical dipole case only. 


\section{APPENDIX D}

\section{GENERALIZED CASE OF AN EARTH WITH CYLINDRICAL SYMMETRY}

Gauss's law in differential form is $\nabla . \mathbf{B}=0$, or equivalently, $\nabla . \mathbf{H}=0$. In circular cylindrical coordinates, the latter equation is (Ward and Hohmann, 1988)

$$
\frac{1}{\rho} \frac{d\left(\rho H_{\rho}\right)}{d \rho}+\frac{1}{\rho} \frac{d H_{\theta}}{d \theta}+\frac{d H_{z}}{d z}=0 .
$$

Using the chain rule for the first term and ignoring the second term, because the cylindrical symmetry means that there is no variation in the $\theta$ direction, results in

$$
\frac{1}{\rho} H_{\rho}+\frac{d H_{\rho}}{d \rho}+\frac{d H_{z}}{d z}=0 .
$$

The coordinate of the airborne EM system assumes that $z$ is directed upward, so

$$
\frac{1}{\rho} H_{\rho}+\frac{d H_{\rho}}{d \rho}-\frac{d H_{z}}{d z}=0
$$

Assuming $B=\mu H$, the right-hand-side can be shown to be $\Delta_{L}$ in equation A-7.

The differential form of Faraday's law of induction is

$$
\nabla \times \mathbf{H}=\sigma \mathbf{E}+\varepsilon \frac{d \mathbf{E}}{d t}
$$

The $\theta$ component of $\nabla \times \mathbf{H}$ is

$$
\frac{d H_{\rho}}{d z}-\frac{d H_{z}}{d \rho}=\sigma E_{\theta}+\varepsilon \frac{d E_{\theta}}{d t} .
$$

At the receiver, $\sigma=0$ and we make the quasi-static assumption $\varepsilon=0$, so with an appropriate modification due to the $z$-direction being reversed, gives

$$
\frac{d H_{\rho}}{d z}+\frac{d H_{z}}{d \rho}=0 .
$$

Once again, the right-hand-side can be equated with $\Delta_{X}$ in equation A-8.

The other components of $\nabla \times \mathbf{H}$ do not provide any other relations between gradient components, because the cylindrical symmetry removes the components associated with $\theta$ derivatives.

\section{REFERENCES}

Abramowitz, M., and I. A. Stegun, 1965, Handbook of mathematical functions: Dover.

Adams, C., J. Macnae, A. Davis, Y. Ley-Cooper, and A. Smiarowski, 2010, Remote resistivity contrast mapping using the CARIS system at Terme de Caracalla archaeological site, Rome, Italy: Near Surface Geophysics, 8 , 195-202, doi: 10.3997/1873-0604.2010003.

Allard, M., 2007, On the origin of the HTEM species, in B. Milkereit, ed., Proceedings of Exploration 07: Fifth decennial international conference on mineral exploration: Decennial Mineral Exploration Conferences, 535-374

Annan, A. P., 1974, The equivalent source method for electromagnetic scattering analysis and its geophysical application: Ph.D. thesis, Memorial University of Newfoundland.

Boivin, M., 2007, Advances in geophysical technology for VMS exploration, in B. Milkereit, ed., Proceedings of exploration 07: Fifth decennia international conference on mineral exploration: Decennial Mineral Exploration Conferences, 731-739.

Cheng, L. Z., R. S. Smith, M. Allard, P. Keating, M. Chouteau, J. Lemieux M. A. VallØe, D. Bois, and D. Fountain, 2006, Geophysical case study of the Iso and New Insco deposits, Quebec, Canada: Part I, Data comparison and analysis: Exploration and Mining Geology: Journal of the Geological Society of CIM, 15, 53-63, doi: 10.2113/gsemg.15.1-2.53.

Dransfield, M., 2007, Airborne gravity gradiometry in the search for mineral deposits: in B. Milkereit, ed., Proceedings of exploration 07: Fifth decennial international conference on mineral exploration: Decennial Mineral Exploration Conferences, 341-354.

Hood, P. J., and D. J. Teskey, 1989, Aeromagnetic gradiometer program of the Geological Survey of Canada: Geophysics, 54, 1012-1022, doi: 10 $.1190 / 1.1442726$.

Kolaj, M., and R. Smith, 2012, Using spatial gradients of electromagnetic data to map lateral variations in mine tailings: 82nd Annual International Meeting, SEG, Expanded Abstracts, 1-5.

Lee, J. B., 2010, Feasibility of in-phase airborne TEM utilising gradient measurements: ASEG, Extended Abstracts, 1-4.

Macnae, J. C., 1981, Geophysical prospecting with electric fields from an inductive EM source: Ph.D. thesis, University of Toronto.

Murray, I. R., C. Alvarez, and R. W. Groom, 1999, Modelling of complex electromagnetic targets using advanced non-linear approximator techniques: 69th Annual International Meeting, SEG, Expanded Abstracts, 271-274.

Sattel, D., and J. C. Macnae, 2001, The feasibility of electromagnetic gradiometer measurements: Geophysical Prospecting, 49, 309-320, doi: 10 $.1046 /$ j.1365-2478.2001.00244.x.

Smith, R. S., and A. P. Annan, 1997, Advances in airborne time-domain EM technology: in A. G. Gubins, ed., Proceedings of exploration 97: Fourth decennial international conference on mineral exploration, GEO F/X, 497-504.

Smith, R. S., and A. P. Annan, 2000, Using an induction coil sensor to indirectly measure the $B$-field response in the bandwidth of the transient electromagnetic method: Geophysics, 65, 1489-1494, doi: 10.1190/1 1444837.

Smith, R. S., G. Hodges, and J. Lemieux, 2009, Case histories illustrating the characteristics of the HeliGEOTEM system: Exploration Geophysics, 40, 246-256, doi: 10.1071/EG09006.

Spies, B. R., 1975, The dual loop configuration of the transient electromagnetic method: Geophysics, 40, 1051-1057, doi: 10.1190/1.1440581.

Telford, W. M., L. P. Geldart, R. E. Sheriff, and D. A. Keys, 1976, Applied geophysics: Cambridge University Press.

van Leeuwen, E. H., 2000, BHP develops airborne gravity gradiometer for mineral exploration: The Leading Edge, 19, 1296-1297, doi: 10.1190/1 .1438526.

Vozoff, K., 1991, The magnetotelluric method: in M. N. Nahbighian, ed., Electromagnetic methods in applied geophysics vol. 2, Applications: SEG, 641-712.

Ward, S. H., and G. W. Hohmann, 1988, Electromagnetic theory for geophysical applications: in M. N. Nahbighian, ed., Electromagnetic methods in applied geophysics vol. 1, Theory: SEG, 130-311. 